Pacific Journal of Mathematic 


\title{
APPROXIMATE IDENTITIES AND THE STRICT TOPOLOGY
}

\author{
H. S. Collins AND R. A. Fontenot
}

This paper studies relationships between approximate identities on a $B^{*}$ algebra $A$ and other properties of the algebra. If $A$ is commutative, conditions on the approximate identity for $A$ are related to topological properties of the spectrum of $A$. The principal result of this paper is that for a locally compact Hausdorff space $S, C_{0}(S)$ has an approximate identity that is totally bounded in the strict topology (or compact open topology) if and only if $S$ is paracompact.

1. Introduction. The problem of extending theorems about commutative $B^{*}$ algebras to the non-commutative case has received a great deal of attention in recent years. Because many proofs made in the commutative case make use of the spectrum (= maximal ideal space), an obvious question is: what is to replace this device in the case of a non-commutative $B^{*}$ algebra? Various possible replacements have been sought; e.g.; see Akemann [1] and Pedersen [15, 16]. Much progress has been made for certain types of problems by means of restrictions on approximate identities for the algebra in question by Taylor [20, 21], Akemann [2], and others. The class of problems solved or seemingly susceptible to this technique is rather large. This fact and the paucity of results for this class of problems obtained by studying Prim $A$ and the space of equivalence classes of irreducible representations suggest that the approximate identity is a useful tool for extending many commutative theorems to a nonabelian setting. A question that arises immediately in the case of a commutative $B^{*}$ algebra is: what do restrictions on the approximate identity imply about the spectrum of $A$ and vice versa? Along this line, Collins-Dorroh [6] characterize $\sigma$-compactness of the spectrum and ask for necesary and sufficient conditions on $S$ that $C_{0}(S)$ (in this paper, $S$ always denotes a locally compact Hausdorff space) have an approximate identity that is totally bounded in the strict topology (called $\beta$ by Buck). This paper answers this question and several related ones, including some in the non-commutative context.

\section{Preliminaries.}

DEFINITION 2.1. Let $A$ be a Banach algebra. An approximate identity for $A$ is a net $\left\{e_{\lambda} \mid \lambda \in \Lambda\right\}$ (we generally write simply $\left\{e_{\lambda}\right\}$ ) with $\lim _{\lambda}\left\|e_{\lambda} x-x\right\|=\lim _{2}\left\|x e_{2}-x\right\|=0$ for $x \in A$ and $\left\|e_{\lambda}\right\| \leqq 1$ for all $\lambda$. 
It is well known that all $B *$ algebras have approximate identities.

DEFINITION 2.2. The double centralizer algebra $M(A)$ of a $B^{*}$ algebra $A$ was studied by R. C. Busby [5] who defined the strict topology as that topology on $M(A)$ generated by the seminorms $x \rightarrow$ $\max \{\|x y\|,\|y x\|\}$ for $x \in M(A)$ and $y \in A$. Two motivating examples for the double centralizer algebra concept are the algebra $C_{0}(S)$ of continuous complex functions on $S$ which vanish at infinity (this class is identical with the class of all commutative $C^{*}$ algebras by the theorem of Gelfand), whose double centralizer algebra was identified by Wang [22] as $C_{b}(S)$, the algebra of all bounded continuous complex functions on $S$; and the algebra of compact operators on a Hilbert space $H$, whose double centralizer algebra was shown to be the bounded linear operators on $H$ by Busby. For a definition of $M(A)$ and some of its properties, the reader is referred to Busby [5]. By $M(A)_{\beta}$ we shall mean $M(A)$ endowed with the strict topology $\beta$.

Definition 2.3. If $f \in C_{b}(S)$, the support of $f$, spt $f$, is the closure in $S$ of $N(f)=\{x: f(x) \neq 0\}$.

Definition 2.4. $S$ is sham compact if each $\sigma$-compact subset is relatively compact.

Definition 2.5. Let $A$ be a $B^{*}$ algebra and $\left\{e_{\lambda}\right\}$ be an approximate identity for $A$. We shall be interested in the following conditions:

(a) $\left\{e_{\lambda}\right\}$ is countable, i.e., the range of $\left\{e_{\lambda}\right\}$ is a countable set;

(b) $\left\{e_{\lambda}\right\}$ is sequential, i.e., $\Lambda$ is the set of positive integers with the usual order;

(c) $\left\{e_{\lambda}\right\}$ is canonical, i.e., $e_{\lambda} \geqq 0$ and if $\lambda_{1}<\lambda_{2}$ then $e_{\lambda_{1}} e_{\lambda_{2}}=e_{\lambda_{1}}$;

(d) $\left\{e_{\lambda}\right\}$ is well-behaved (after Taylor [21]), i.e., $\left\{e_{\lambda}\right\}$ is canonical and if $\lambda \in \Lambda$ and $\left\{\lambda_{n}\right\}$ is a strictly increasing sequence in $\Lambda$, there is a positive integer $N$ so that $e_{\lambda} e_{\lambda_{n}}=e_{\lambda} e_{\lambda_{m}}$ for $n, m>N$;

(e) $\left\{e_{\lambda}\right\}$ is $\beta$ totally bunded; i.e., totally bounded in the strict topology;

(f) $\left\{e_{\lambda}\right\}$ is abelian;

(g) $\left\{e_{\lambda}\right\}$ is chain totally bounded, i.e., if $\left\{\lambda_{n}\right\}$ is an increasing sequence in $\Lambda$, then $\left\{e_{\lambda_{n}}\right\}$ is $\beta$ totally bounded;

(h) $\left\{e_{\lambda}\right\}$ is $\sigma\left(M(A), M(A)_{\beta}^{*}\right)$ relatively compact, where $\sigma$ denotes the weak topology on $M(A)$ in the pairing with its $\beta$ dual;

(i) $\left\{e_{\lambda}\right\}$ is sham compact, i.e., $\left\{e_{\lambda}\right\}$ is canonical and if $\left\{\lambda_{n}\right\}$ is a sequence in $\Lambda$, then there is a $\lambda$ in $\Lambda$ so that $\lambda>\lambda_{n}$ for all integers $n$.

REMARK 2.6. A sequence $\left\{e_{n}\right\}$ in a $B^{*}$ algebra $A$ which satisfies 
$\lim _{n}\left\|e_{n} x-x\right\|=\lim _{n}\left\|x e_{n}-x\right\|=0$ is norm bounded by the uniform boundedness principle and the $B *$ norm property. Thus it is not necessary to require norm boundedness in 2.1 for this case.

REMARK 2.7. Taylor [21] introduced the notion of a well-behaved approximate identity and used it to prove many interesting improvements of results of Phillips [9, p. 32], Akemann [2], Bade [3], CollinsDorroh [6], and Conway [7 and 8].

3. A characterization of paracompact spaces. Our main result in this section, 3.10, answers two questions posed in [6, Remark 4.3]. Our interest centers exclusively on $B^{*}$ algebras without identity; for these, we need information about increasing sequences in the directed set of an appropriate identity and about supports. Lemmas 3.1 and 3.2 provide what we need.

LEMma 3.1. If $A$ is a Banach algebra without identity, $\left\{e_{\lambda}\right\}$ an approximate identity for $A$, and $\lambda_{0} \in \Lambda$, then $\exists \lambda \in \Lambda \cdot \ni \cdot \lambda>\lambda_{0}$.

Proof. If the conclusion does not hold, then $\forall \lambda \in \Lambda, \lambda \leqq \lambda_{0}$, from which it follows that $e_{\lambda_{0}}$ is an identity for $A$.

LEMMA 3.2. Let $\left\{e_{\lambda}\right\}$ be an approximate identity for $C_{0}(S)$.

(a) If $\left\{e_{\lambda}\right\}$ is canonical, then $\lambda_{1}<\lambda_{2}$ implies spt $e_{\lambda_{1}} \subset e_{\lambda_{2}}^{-1}\{1\} \subset$ $N\left(e_{\lambda_{2}}\right)$ and $\lambda \in \Lambda$ implies that the spt $e_{\lambda}$ is compact;

(b) If $K$ is a compact subset of $S$, then $\exists \lambda \in \Lambda$ so that $\left|e_{\lambda}\right|>3 / 4$ on $K$.

\section{Proof. This is straightforward.}

We are mainly interested (in $\S 3$ ) in two types of approximate identities, viz., well-behaved ones, shown to be important by Taylor [21], and $\beta$ totally bounded ones, the study of which motivated this paper.

LEMMA 3.3. Let $\left\{e_{\lambda}\right\}$ be an approximate identity for $C_{0}(S)$ which is either $\beta$ totally bounded or well-behaved. Then there exists a cover of $S$ by clopen $\sigma$-compact sets.

REMARK 3.4. All topologies between the compact open and the strict agree on norm bounded sets. Thus " $\beta$ totally bounded" may be replaced in 3.3 by "compact open totally bounded."

Proof of 3.3. We assume that $S$ is not compact in either case to 
avoid trivialities. Assume first that $\left\{e_{\lambda}\right\}$ is $\beta$ totally bounded. Replacing $\left\{e_{\lambda}\right\}$ by $\left\{\left|e_{\lambda}\right|^{2}\right\}$, performing a straight forward computation and using 3.4, we may assume that $\left\{e_{\lambda}\right\}$ is compact open totally bounded and $e_{\lambda} \geqq 0$ for each $\lambda$. Let $x \in X$ and choose by 3.2 (b) $\lambda_{1} \in \Lambda$ so that $e_{\lambda_{1}}(x)>3 / 4$. Let $K_{1}=\left\{x \in S: e_{\lambda_{1}}(x) \geqq 1 / 4\right\}$. Suppose that $\left\{K_{j}\right\} j=1, \cdots, n$ and $\left\{\lambda_{j}\right\} j=1, \cdots, n$ have been chosen so that

$$
\begin{gathered}
e_{\lambda_{j}}>\frac{3}{4} \text { on } K_{j-1}, \quad j=1, \cdots, n \\
K_{j}=\left\{x \in S: e_{\lambda_{i}}(x) \geqq \frac{1}{4 j} \text { for some } i, 1 \leqq i \leqq j\right\} .
\end{gathered}
$$

By 3.2 (b) again, choose $\lambda_{n+1} \in \Lambda$ so that $e_{\lambda_{n+1}}>3 / 4$ on $K_{n}$ and let

$$
K_{n+1}=\left\{x \in S: e_{\lambda_{i}}(x) \geqq \frac{1}{4^{n+1}} \quad \text { for some } \quad i, 1 \leqq i \leqq n+1\right\} .
$$

By induction we obtain sequences $\left\{\lambda_{n}\right\}$ and $\left\{K_{n}\right\}$ satisfying (1) and (2) above. Let $X=\bigcup_{n} K_{n}$. $X$ is clearly $\sigma$-compact and contains $x$. It is open since $K_{n} \subset$ interior of $K_{n+1}$. To show that $X$ is closed, take a compact set $K$. It suffices to show $K \cap X$ is closed [13, p. 231]. The total boundedness condition of $\left\{e_{\lambda}\right\}$ gives the existence of an integer $i_{0}$ so that for all positive integers $j$,

$$
\min _{1 \leqq i \leqq i_{0}}\left\|e_{\lambda_{j}}-e_{\lambda_{i}}\right\|_{K}<\frac{1}{4}
$$

$\left(\|f\|_{K}=\sup _{x \in K}|f(x)|\right.$ for $\left.f \in C_{b}(S)\right)$. Let $y \in K_{m} \cap K$ where $m>i_{0}$. By construction $e_{\lambda_{m+1}}(y)>3 / 4$ so by (3) there is an integer $1 \leqq i \leqq i_{0}$ so that $e_{\lambda_{i}}(y) \geqq 1 / 2$ which shows that $y \in K_{i}$. Thus $X \cap K=K \cap \bigcup_{i=1}^{i_{0}} K_{i}$ so $X \cap K$ is closed.

For the other part of the lemma, let $x \in X$, assume that $\left\{e_{\lambda}\right\}$ is well-behaved, and choose by 3.1 and 3.2 an increasing sequence $\left\{\lambda_{n}\right\}$ so that $e_{\lambda_{1}}(x)>0$. Let $K_{n}=$ spt $e_{\lambda_{n}}$ and note, by 3.2 , that $K_{n} \subset$ interior of $K_{n+1}$. Let $X=\cup K_{n}$ and note that $X$ is open, $\sigma$-compact and $x \in X$. From 3.2 (a) and the definition of well-behaved approximate identity, it follows that $\left\{e_{\lambda_{i}}\right\}$ is totally bounded in the compact open topology and that $y \in X$ implies $e_{\lambda_{j}}(y)=1$ for $j$ large enough. With these observations, the proof that $X$ is closed is the same as in the first part of the lemma.

REMARK 3.5. Note that in 3.3, $\mathrm{U}_{n=1}^{\infty}$ spt $e_{\lambda_{n}} \subset X$.

COROLlaRY 3.6. If $S$ is connected and has an approximate identity that is either well-behaved or $\beta$ totally bounded, then $S$ is $\sigma$ compact. 
Proposition 3.7. Let $F$ be a closed subset of $S$. If $C_{0}(S)$ has either a well-behaved or a $\beta$ totally bounded approximate identity, then $F$ contains a $\sigma$-compact set that is relatively clopen in $F$.

Proof. Let $\left\{e_{\lambda}\right\}$ be an approximate identity with either of the properties above. For $\lambda \in \Lambda$, let $d_{\lambda}$ be the restriction of $e_{\lambda}$ to $F$. Since $F$ is closed, $\left\{d_{\lambda}\right\} \subset C_{0}(F)$. We claim that $\left\{d_{\lambda}\right\}$ has the same property as $\left\{e_{\lambda}\right\}$ does; i.e., that $\left\{d_{\lambda}\right\}$ is a well-behaved (resp. $\beta$ totally bounded) approximate identity for $C_{0}(F)$. To show this, it suffices to show that if $f \in C_{0}(F)$, then there is an extension $g$ in $C_{0}(S)$ of $f$. Let $S^{*}$ denote the one-point compactification of $S$ and $\infty$ denote the point at infinity. Let $f^{\prime}$ be an extension of $f$ to $F \cup\{\infty\}$ obtained by defining $f^{\prime}(\infty)=0$. Since $f \in C_{0}(F), f^{\prime}$ is continuous and extends to a continuous function $p$ on all of $S^{*}$ by Tietze's Theorem since $F \cup\{\infty\}$ is closed in $S^{*}$. The restriction $g$ of $p$ to $S$ is clearly an extension of $f$ in $C_{0}(S)$. This concludes the proof of 3.7 .

CoRollary 3.8. If $S$ is locally connected and $C_{0}(S)$ has an approximate identity that is either well-behaved or $\beta$ totally bounded, then $S$ is paracompact.

Proof. By [11, Theorem 7.3], it suffices to show that $S$ is a disjoint union of clopen $\sigma$-compact subspaces. In a locally connected space, the components are clopen and connected and so $\sigma$-compact by 3.7.

Lemma 3.9. Suppose that $C_{0}(S)$ has a $\beta$ totally bounded approximate identity and let $\mathscr{W}$ be the family of all clopen $\sigma$-compact subsets of $S$ constructed by the method of the first part of 3.3. If $\mathscr{C} \subset \mathscr{W}$, then $\bigcup_{W \in \mathscr{H}} W$ is clopen.

Proof. We may assume $e_{\lambda} \geqq 0$ as in 3.3. Let $X=\bigcup_{W \in \ddot{H}} W$ and $K$ be an arbitrary compact subset of $S$. Since $S$ is locally compact, it suffices to show that $X \cap K$ is closed. With each $W$ in $\mathscr{C}$ is associated a sequence $\left\{e_{n}^{W}\right\}$ from the approximate identity such that

$$
\bigcup_{n=1}^{\infty} \operatorname{spt} e_{n}^{W} \subset W
$$

(see 3.4) and if $\gamma \in W, e_{n}^{W}(\gamma)>3 / 4$ for $n$ large enough. From $\beta$ total boundedness of $\left\{e_{n}^{W}: W \in \mathscr{L}, n=1,2, \cdots\right\}$, we get a set $\left\{W_{i}\right\}_{i=1, \ldots, n}$ from $\mathscr{Q}$ and associated integers $\left\{n_{i}\right\} i=1, \cdots, n$ so that for any $V$ in $\mathscr{C}$ and positive integer $p$

$$
\min _{1 \leqq i \leqq n}\left\|e_{n_{i}}^{W}-e_{p}^{V}\right\|_{K}<\frac{1}{4} .
$$


If $\gamma \in X \cap K$, then $\gamma \in K \cap W$ for some $W \in \mathscr{L}$, so choosing $p$ large enough so that $e_{p}^{V}(\gamma)>3 / 4$ we see that $e_{n_{i}}^{W}(\gamma)>0$ for some $1 \leqq i \leqq n$ so that $\gamma \in W_{i}$. We have established that $X \cap K=K \cap \bigcup_{i=1}^{n} W_{i}$ so $X \cap K$ is closed. This concludes the proof of 3.9 .

In [6] Collins and Dorroh show that if $S$ is paracompact then $C_{0}(S)$ has a $\beta$ totally bounded approximate identity and ask two questions: (1) Does the existence of a $\beta$ totally bounded approximate identity imply the existence of a canonical one that is $\beta$ totally bounded? and (2) Does the existence of a $\beta$ totally bounded approximate identity in $C_{0}(S)$ imply that $S$ is paracompact? We add to these a third question: Does the existence of a $\beta$ totally bounded approximate identity in $C_{0}(S)$ imply the existence of a well-behaved one? The answer to all these questions is given in 3.10 .

THEOREM 3.10. These are equivalent: (1) $S$ is paracompact; (2) $C_{0}(S)$ has a canonical approximate identity that is $\beta$ totally bounded; (3) $C_{0}(S)$ has a approximate identity that is $\beta$ totally bounded.

Proof. For the first implication see [6]. Since the second implication is trivial, we prove only that if $\left\{e_{\lambda}\right\}$ is a $\beta$ totally bounded approximate identity for $C_{0}(S)$ then $S$ is paracompact. Take $\mathscr{W}$ to be the set in 3.9 and well order it. Let $W_{0}$ be the first element in $\mathscr{W}$ and $W_{0}^{\prime}=W_{0}$. If $W \in \mathscr{P}$, and $W \neq W_{0}$, let $W^{\prime}=W \backslash\left(\bigcup_{V \in W}^{V<W}\right.$,

Each set $W^{\prime}$ is clopen and $\sigma$-compact by 3.3 and 3.9 .

If $x \in S$ and $W$ is the least element in $\{W: W \in \mathscr{W}$ and $x \in W\}$, then $x$ clearly belongs to $W^{\prime}$. The collection $\left\{W^{\prime}: W \in \mathscr{W}\right\}$ then consists of disjoint sets and so forms a partition of $S$ by clopen $\sigma$-compact subsets. We apply [11, Theorem 7.3] to conclude the proof.

4. Non-commutative results and examples. Taylor [21] gives the following examples of $B^{*}$ algebras with well-behaved approximate identities: algebras with countable approximate identities, algebras with series approximate identities (for a definition, see Akemann [23]) such as the compact operators on a Hilbert space, and subdirect sums of algebras having well-behaved approximate identities, such as dual $B^{*}$ algebras which are subdirect sums of algebras of compact operators.

In this section, we give examples of algebras with $\beta$ totally bounded approximate identities using some techniques borrowed from Taylor and some of our own. We also give some partial results, e.g., 4.1, relating the existence of approximate identities of one type to existence of another type. 
Proposition 4.1. Let $A$ be a Banach algebra with a sequential canonical approximate identity $\left\{e_{n}\right\}$. Then $\left\{e_{n}\right\}$ is $\beta$ totally bounded and well behaved.

The proof requires the following observation whose proof is straightforward:

REMARK 4.2. If $\left\{f_{\lambda}\right\}$ is an approximate identity for $A$, then the locally convex topology on $M(A)$ (see 2.2) generated by the seminorms $x \rightarrow \max \left\{\left\|f_{\lambda} x\right\|,\left\|x f_{\lambda}\right\|\right\}$ agrees with the strict topology on norm bounded sets in $M(A)$.

Proof of 4.1. Let $m$ and $n_{1}<n_{2}<\cdots$ be positive integers. Choose a positive integer $i_{0}$ so that $n_{i}>m$ for $i \geqq i_{0}$. Then

$$
e_{m}\left(e_{n_{i}}-e_{n_{j}}\right)=0
$$

for $i, j>i_{0}$ by the canonical property so $\left\{e_{n}\right\}$ is well-behaved. Total boundedness in the strict topology follows from 4.2 and the fact that $\left\{e_{n}\right\}$ is well-behaved. Part (a) of the next result was used by Taylor [21] in his study of well-behaved identities. We shall use it in 4.5 to show that algebras with countable approximate identities have ones with other nice properties.

LEMma 4.3. Let $A$ be a Banach algebra. (a) If $\left\{e_{\lambda}\right\}$ is an approximate identity for $A$ and $\left\{f_{p}\right\}$ is an approximate identity for the normed algebra generated by $\left\{e_{\lambda}\right\}$, then $\left\{f_{p}\right\}$ is an approximate identity for $A$; (b) If $\left\{e_{\lambda}\right\}$ is a norm bounded net in $A$ and $D$ a dense subset in the Hermitian part of the unit ball of $A$ so that $e_{\lambda} x \rightarrow x$ and $x e_{\lambda} \rightarrow x$ for each $x$ in $D$, then $\left\{e_{\lambda}\right\}$ is an approximate identity for $A$ (here we assume $A$ is $B^{*}$ ).

Proof. This is a straightforward computation.

Separable $B^{*}$ algebras have many types of approximate identities as 4.4 shows.

Lemma 4.4. Let $A$ be a separable $B^{*}$ algebra. Then $A$ contains an approximate identity that is canonical, sequential, and abelian (and by 4.1, well-behaved and $\beta$ totally bounded).

Proof. Let $\left\{x_{n}\right\}$ be a countable dense set in the Hermitian part of the unit sphere of $A$, and let $x=\sum\left(1 / 2^{n}\right) x_{n}^{2}$. Since $x$ is a positive element of $A$, the $B^{*}$ algebra $C$ generated by $x$ is isometrically *-isomorphic to the algebra $C_{0}(S)$, where $S$ is the maximal ideal space of 
$C$. Since $C_{0}(S)$ is generated by a single function, $S$ is $\sigma$-compact. We may select from $C\left(=C_{0}(S)\right)$ an approximate identity $\left\{e_{k}\right\}$ for $C$ possessing all the properties mentioned in the statement of 4.4. It remains only to show that $\left\{e_{k}\right\}$ is an approximate identity for $A$. Adjoin a unit $I$ to $A$ in the customary manner so that the adjoined algebra is $B^{*}$, hence we have that $\left\|\left(I-e_{k}\right) x\left(I-e_{k}\right)\right\| \underset{l_{k}}{\rightarrow} 0$. From $[10, p .14]$ we have that

$$
\left\|\left(I-e_{k}\right) x_{n} x_{n}^{*}\left(I-e_{k}\right)\right\| \leqq 2^{n}\left\|\left(I-e_{k}\right) x\left(I-e_{k}\right)\right\|
$$

so that $\left\|\left(I-e_{k}\right) x_{n}\right\|=\left\|x_{n}\left(I-e_{k}\right)\right\| \rightarrow{ }_{k} 0$. Thus applying 4.3 (b) to $D=$ $\left\{x_{n}\right\}$ and $\left\{e_{k}\right\}$ we see that $\left\{e_{k}\right\}$ is an approximate identity for $A$.

Definition 4.5. Let $\left\{A_{\gamma}\right\}$ be a family of normed algebras. The subdirect sum, $\left(\sum A_{\gamma}\right)_{0}$, of the family $\left\{A_{\gamma}\right\}$ is that subset of $P_{\gamma \in \Gamma} A_{\gamma}$ consisting of all $a=\left(a_{\gamma}\right) \in P A_{\gamma}$ so that $\left\{\gamma \in \Gamma:\left\|a_{\gamma}\right\| \geqq \varepsilon\right\}$ is finite for each $\varepsilon>0$. The algebraic operations are pointwise and $\|a\|=$ $\sup \left\{\left\|a_{r}\right\|: \gamma \in \Gamma\right\}$.

Proposition 4.6. If $A=\left(\sum A_{\gamma}\right)_{0}$ and each $A_{i}$ has a $\beta$ totally bounded approximate identity, then so does $A$.

Proof. The proof is the same as Proposition 3.2 in [21] where the same result is proved for well-behaved approximate identities.

Remark 4.7. Proposition 4.6 is true when "totally bounded" is replaced by any of the types of approximate identities listed in $\S 2$, except countable and sequential. Dual $B^{*}$ algebras have $\beta$ totally bounded approximate identities by 4.6 , and 4.5 and 4.6 give a proof, different from that in [6], that $C_{0}(S)$, for $S$ paracompact, has a $\beta$ totally bounded approximate identity.

Conjecture 4.8. We conjecture that $C_{0}(S)$ has a well-behaved approximate identity if and only if $S$ is paracompact. As we indicated earlier, our results on this question are incomplete, but we give an example in $\S 6$ that is perhaps illuminating.

5. Sham compact spaces and approximate identities. The definition of sham compact space and sham compact approximate identity, given in 2.5 , is motivated by the space $X$ of ordinals less than the first uncountable ordinal with the order topology, and the algebra $C_{0}(X)$. For example, let $\Lambda=X$ with the usual order and if $\lambda \in \Lambda$, let $f_{\lambda}$ be the characteristic function of the interval $[0, \lambda]$. It is clear that $\left\{f_{\lambda}\right\}$ is a sham compact approximate identity for $C_{0}(X)$. We note that $C_{0}(X)$ cannot have a $\beta$ totally bounded approximate 
identity since $X$ is not paracompact. Furthermore, it cannot have a well-behaved approximate identity either since it is pseudocompact.

Proposition 5.1. Let $S$ be pseudocompact. If $C_{0}(S)$ has a wellbehaved approximate identity, then $S$ is compact.

Proof. Let $\left\{e_{\lambda}\right\}$ be a well-behaved approximate identity for $C_{0}(S)$, suppose that $S$ is not compact, and choose, by 3.1, an increasing sequence $\left\{\lambda_{n}\right\}$ so that $e_{\lambda_{i}} \neq e_{\lambda_{i+1}}$ for any integer $i_{\text {o }}$ Note that $e_{\lambda_{1}}<$ $e_{\lambda_{2}}<\cdots$, i.e., $\left\{e_{\lambda_{i}}\right\}$ is an increasing sequence. Since the sequence $\left\{e_{\lambda_{i}}\right\}$ is Cauchy in the compact open topology and $C_{b}(S)$ is complete in this topology, there is a function $f$ in $C_{b}(S)$ so that $e_{\lambda_{i}} \rightarrow f$ uniformly on compact subsets of $S$. By [12, Theorem 2], $e_{\lambda_{i}} \rightarrow f$ in norm so $f$ is in $C_{0}(S)$. By 3.2, $f \equiv 1$ on $\bigcup_{i=1}^{\infty}$ spt $e_{\lambda_{i}}$ which then is contained in the compact set $K=f^{-1}\{1\}$. Choosing $\lambda \in \Lambda$ so that $e_{\lambda} \equiv 1$ on $K$, we obtain a contradiction to the fact that $e_{\lambda_{i}} \neq e_{\lambda_{i+1}}$ for all $i$.

REMARK 5.2. Proposition 5.1 admits the following non-abelian generalization, stated here, without proof, for completeness: Suppose a $B^{*}$ algebra $A$ has a well-behaved approximate identity and $M(A)$ satisfies the following condition: whenever $\left\{a_{n}\right\}$ is an increasing sequence in $A$ and $\left\{a_{n}\right\}$ converges in the strict topology to $x$ in $M(A)$, then $\left\|a_{n}-x\right\| \rightarrow 0$. Then $A$ has an identity and $A=M(A)$. (See [12, Proposition 2] to see that this result includes 5.1.)

The next proposition relates sham compactness of $S$, existence of sham compact approximate identities in $C_{0}(S)$ and the property $(D F)$ of Grothendieck.

DEFINITION 5.3. Let $E$ be a locally convex topological vector space with dual $E^{*}$. The space $E$ is $(D F)$ if there is a countable base for bounded sets in $E$ and if every countable intersection of closed convex circled zero neighborhoods which absorbs bounded sets is a zero neighborhood.

REMARK 5.4. The vector space $C_{b}(S)_{\beta}$ is complete and the $\beta$ bounded sets coincide with the norm bounded sets so $C_{b}(S)_{\beta}$ is $(D F)$ if each countable intersection of closed convex circled zero neighborhoods which absorbs points of $C_{b}(S)$ is a zero neighborhood [17, p. 67].

We shall use the following remark in the proof of Theorem 5.6.

Remark 5.5. W. H. Summers [19] has recently shown that $C_{b}(S)_{\beta}$ 
is $(D F)$ if $C_{b}\left(N ; C_{0}(S)\right)$ is essential, where $C_{b}\left(N ; C_{0}(S)\right)$ is the Banach algebra of all norm bounded sequence from $C_{0}(S)$ with the sup norm topology $\left(\|\|_{\infty}\right)$ and "essential" means that $\left\|e_{\lambda}\left\{f_{n}\right\}-\left\{f_{n}\right\}\right\|_{\infty} \rightarrow_{\lambda} 0$ where $\left\{e_{\lambda}\right\}$ is any approximate identity for $C_{0}(S)$ and $\left\{f_{n}\right\}$ any element of $C_{b}\left(N ; C_{0}(S)\right)$.

Theorem 5.6. These are equivalent: (a) $C_{b}(S)_{\beta}$ is $(D F)$; (b) $S$ is a sham compact space (c) $C_{0}(S)$ has a sham compact approximate identity.

Proof. Assume that $C_{b}(S)_{\beta}$ is $(D F)$ and $X$ is the union of compact sets $K_{n}$, i.e., $X=\bigcup_{n=1}^{\infty} K_{n}$. For each integer $n$, let $\varphi_{n}$ be a function in $C_{0}(S)$ so that $0 \leqq \varphi_{n} \leqq 1$ and $\varphi_{n} \equiv 1$ on $K_{n}$. Let

$$
V=\left\{f \in C_{b}(S):\left\|f \varphi_{n}\right\| \leqq 1, \forall_{n}\right\} .
$$

$V$ absorbs points of $C_{b}(S)$; therefore it is a zero neighborhood in the strict topology by (a). It is obvious that the sets $\left\{f \in C_{b}(S):\|f \varphi\| \leqq 1\right\}$ (for $\varphi \geqq 0$ in $C_{0}(S)$ ) is a base at zero for the strict topology. Thus $\exists \varphi \geqq 0$ in $C_{0}(S)$ so that $\left\{f \in C_{b}(S):\|f \varphi\| \leqq 1\right\} \subset V$. This shows that $\varphi(x) \geqq 1$ for $x$ in $X$. For if not, there is an integer $n$ and a point $x_{0}$ in $K_{n}$ so that $\varphi\left(x_{0}\right)<1$. By a standard Urysohn's lemma argument $\exists f \in C_{0}(S)$ so that $f\left(x_{0}\right)>1$ and $\|\varphi f\|<1$. This contradiction establishes our claim, i.e., $X \subset \varphi^{-1}\{1\}$, so $X$ is compact.

Suppose that (b) holds. Let $\Lambda$ be the set of all pairs $(K, 0)$ where $K \subset D \subset S, K$ is compact and 0 is open with compact closure. If $\lambda=(K, 0)$ and $\lambda_{1}=\left(K_{1}, 0_{1}\right)$, we define $\lambda \geqq \lambda_{1}$ if $\lambda=\lambda_{1}$ or if $0_{1} \subset K$. If $\lambda=(K, 0)$ let $f_{\lambda}$ be a function in $C_{0}(S)$ which satisfies: (1) $0 \leqq f_{\lambda} \leqq$ 1 ; (2) $f_{\lambda} \equiv 1$ on $K$; and (3) $\operatorname{spt} f_{\lambda} \subset 0$. The net $\left\{f_{\lambda}\right\}$ is by (b) a sham compact approximate identity for $C_{0}(S)$.

Assume (c), with $\left\{e_{\lambda}\right\}$ a sham compact approximate identity, and let $\left\{f_{n}\right\}$ be a sequence contained in the unit ball of $C_{0}(S)$, and $\varepsilon>0$. Choose a sequence $\left\{\lambda_{n}\right\}$ from $\Lambda$ so that $\left\|e_{\lambda_{n}} f_{n}-f_{n}\right\|<\varepsilon$ for each integer $n$. Let $\lambda_{0} \in A$ be such that $\lambda_{0}>\lambda_{n}$ for all integers $n$. Remark 5.5 and the following computation finish the proof;

$$
\begin{aligned}
\lambda>\lambda_{0} \text { implies }\left\|e_{\lambda} f_{n}-f_{n}\right\| & =\left\|\left(1-e_{\lambda}\right) f_{n}\right\| \\
& =\left\|\left(1-e_{\lambda_{n}}\right)\left(1-e_{\lambda}\right) f_{n}\right\| \\
& \leqq\left\|\left(1-e_{\lambda_{n}}\right) f_{n}\right\|<\varepsilon \text { for all } n .
\end{aligned}
$$

6. Metacompact spaces-an example. We have been unable to prove our conjecture that $S$ is paracompact if $C_{0}(S)$ has a well-behaved approximate identity except in special cases (see $\S 3$ ), but we are able to give an example that shows that metacompactness is not sufficient for existence of a well-behaved approximate identity. 
ExAMPLE 6.1. Let $I$ be the unit interval with the discrete topology and $I^{*}$, the one-point compactification of $I$, with $\infty$ denoting the point at infinity. Similarly, let $N$ denote the positive integers with discrete topology, $N^{*}$ the one-point compactification of $N$, and $w$ the point at infinity. Let $S=I^{*} \times N^{*} \backslash\{(\infty, w)\}$. Being an open set in a compact Hausdorff space, $S$ is locally compact Hausdorff.

To show that $X$ is metacompact, take an open cover $\mathscr{U}$ of $X$. For each point $(\infty, n)$, there is a finite set $F_{n}$ of $I$ so that a member of $\mathscr{Q}$ contains the open set $U_{n}=\left\{(x, n): x \notin F_{n}\right\}$. Similarly, for each point $(x, w)$ there is a finite set $G_{X}$ of $N$ with a member of $\mathscr{C}$ containing the open set $W_{x}=\left\{(x, n): n \notin G_{x}\right\}$. If $(x, y) \in X$ and $x \neq \infty$ and $y \neq w,(x, y)$ is discrete. Let $W_{x, y}=\{(x, y)\}$. It is easily checked that the sets $\left\{W_{x}\right\},\left\{U_{n}\right\}$, and $\left\{W_{x, y}\right\}$ from a point-finite open refinement of $\mathscr{C}$. Recalling that a space is metacompact if each open cover has a point-finite open refinement, we see that $X$ is metacompact.

Before we show that $C_{0}(X)$ has no well-behaved approximate identity, we point out that $X$ is not pseudocompact; thus we cannot simply apply 5.1. In our demonstration that $C_{0}(X)$ does not have a well-behaved approximate identity, we first exhibit a $\left.\sigma(M(X)), C_{b}(X)\right)$ convergent sequence $\left\{\mu_{n}\right\}$ which is not tight, where a subset $H$ of $M(X)$ is tight and if it is bounded and for each $\varepsilon>0$ there is a compact set $K_{\varepsilon}$ in $X$ so that $|\mu|\left(X \backslash K_{\varepsilon}\right)<\varepsilon$ for all $\mu \in H(|\mu|$ denotes the total variation of $\mu$ ). We may then apply corollary 3.4 in [21] to conclude that $C_{0}(X)$ does not have a well-behaved approximate identity.

For each positive integer $n$, let $\mu_{n}$ be the member of $M(X)$ defined by the equation $\mu_{n}(f)=f((\infty, n))-f((\infty, n+1))$ for $f$ in $C_{b}(X)$. Note that the total variation of $\mu_{n}$ satisfies the equation $\left|\mu_{n}\right|(f)=f((\infty, n))+f((\infty, n+1))$ for $f$ in $C_{b}(X)$ and so $\left\|\mu_{n}\right\| \leqq 2$ for each integer $n$. We now show that $\mu_{n} \rightarrow 0$ in the weak-* topology of $M(X)$. Let $f \in C_{b}(X)$ and $n \in N$. Since $f$ is continuous at $(\infty, n)$, for each $\varepsilon>0$, there is a finite subset $I_{s, n}$ of $I$ so that if $x \notin I_{\varepsilon, n},|f(x, n)-f(\infty, n)|<\varepsilon$. Thus there is a countable subset $I_{n}$ of $I$ so that if $x \in I_{n}, f(x, n)=f(\infty, n)$. If $I_{f}$ is the union of the sets $\left\{I_{n}\right\}$, we see that it is countable and if $x \notin I_{f}$ then $f(x, n)=f(\infty, n)$ for all integers $n$. Choose a point $x_{f} \notin I_{f}$. Then the sequence $\left\{\left(x_{f}, n\right)\right\}$ converges to the point $\left(x_{f}, w\right)$ so that $f\left(\left(x_{f}, n\right)\right) \rightarrow f\left(\left(x_{f}, w\right)\right)$. Thus

$$
\lim _{n} f((\infty, n))=\lim _{n} f\left(\left(x_{f}, n\right)\right)=f\left(\left(x_{f}, w\right)\right)
$$

so that

$$
\lim _{n} f((\infty, n))-f((\infty, n+1))=0, \text { i.e., } \mu_{n}(f) \rightarrow 0 .
$$

Since $f$ is arbitrary, we have shown that $\mu_{n} \rightarrow 0$ weak-*. 
We next see that $\left\{\mu_{n}\right\}$ cannot be tight: let $\varepsilon=1 / 2$ and note that a compact set in $X$ can contain only finitely many of the points $(\infty, n)$. If $K$ is a compact subset of $X$ and $(\infty, p) \notin K$, we can choose $f \in$ $C_{b}(X)$ so that spt $f$ is compact, $f((\infty, p))=1, f \equiv 0$ on $K$, and $0 \leqq$ $f \leqq 1$, i.e., so that $\left|\mu_{p}\right|(X \backslash K) \geqq\left|\mu_{p}(f)\right| \geqq|f((\infty, p))|=1$. Applying [21, Cor. 3.4.], we see that $C_{0}(X)$ does not have a well-behaved approximate identity (note that $X$ is not paracompact by [20, 3.1 and $3.2])$.

REMARK 6.2. The space $C_{b}(X)_{\beta}$ where $X$ is as in 6.1 is interesting for several other reasons. First $C_{b}(X)_{\beta}$ is not a strong Mackey space (see [7] for a definition). Conway in [7] has shown that $C_{b}(X)$ is strong Mackey if $X$ is paracompact. The problem of finding topological conditions on $X$ necessary and sufficient for $C_{b}(X)_{\beta}$ to be a strong Mackey (or Mackey) space is an intriguing problem. If we let $\mu_{n}$ be the element of $M(X)$ whose value at $f$ in $C_{b}(X)$ is $f((\infty, n))$, arguments similar to the above show that $\left\{\mu_{n}\right\}$ is weak* Cauchy but has no weak-* limit in $M(X)$, i.e., $M(X)$ is not weak-* sequentially complete (see [6, 5.1]). $C_{b}(X)_{\beta}$ is also not sequentially barrelled (see [23]).

\section{Miscellaneous remarks.}

REMARK 7.1. It is easy to show that if $\left\{e_{\lambda}\right\}$ is a sham compact approximate identity for a (possibly non-abelian) Banach algebra $A$, then $\left\{e_{\lambda}\right\}$ cannot be well-behaved unless $A$ has an identity. The question one really wants to answer is whether $A$ can have another approximate identity that is well-behaved unless $A$ has an identity element. If $A$ is commutative, the question is answered in the negative by 5.1 and 5.6 of this paper. We have the following generalization of Theorem 4.1 in [19]:

THEOREM 7.2. These are equivalent: (1) $M(A)_{\beta}$ is $(D F)(2) M(A)_{\beta}$ is $(W D F)(3) l^{\infty}(A)$ is both a right and a left essential module $\left(l^{\infty}(A)\right.$ is the set of all bounded sequences in $A ; l^{\infty}(A)$ is a right essential module means that if $\left\{f_{\lambda}\right\}$ is any approximate identity for $A$ and $x=$ $\left\{x_{n}\right\} \in l^{\infty}(A)$ then $\left.\lim _{\lambda}\left(\sup _{n}\left\|x_{n} f_{\lambda}-x_{n}\right\|\right)=0\right)$.

Proposition 7.3. Let $A$ have a well-behaved approximate identity and suppose that $\left\{e_{\lambda}\right\}$ is a sham compact approximate identity for $A$. Then $A$ has an identity.

Proof. $x=\left(x_{n}\right) \in l^{\infty}(A)$; we can choose, by induction, a sequence $\left\{\lambda_{k}\right\}$ from $\Lambda$ so that 


$$
\lim _{k}\left\|e_{\lambda_{k}} x_{n}-x_{n}\right\|=\lim _{k}\left\|x_{n} e_{\lambda_{k}}-x_{n}\right\|=0
$$

for all positive integers $n$. By the sham compact property, choose $e_{\lambda}$

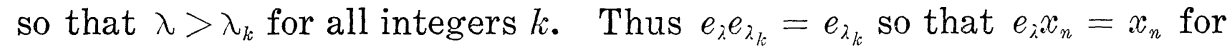
all $n$. Thus $\lim _{\lambda}\left(\sup \left\|x_{n} e_{\lambda}-x_{n}\right\|\right)=0$ and $\lim _{\lambda}\left(\sup _{n} \| e_{\lambda} x_{n}-x_{n}\right)=0$, i.e., $l^{\infty}(A)$ is both left and right essential. Suppose $\left\{f_{r}\right\}$ is a wellbehaved approximate identity for $A$ and $\gamma_{1}<\gamma_{2}<\ldots$ is a sequence in $\Gamma$ so that $0 \neq f_{r_{i}} \neq f_{r_{i+1}}$ for all integers $i$. Since $l^{\infty}(A)$ is essential, there is an element $\gamma_{0}$ is $\Gamma$ so that

$$
\left\|f_{\gamma_{c}} f_{\gamma_{i}}-f_{\gamma_{i}}\right\|<\frac{1}{4}
$$

for all positive integers $i$. Since $\left\{f_{\gamma}\right\}$ is well-behaved, there is a positive integer $N$ so that $n, m \geqq N$ implies that

$$
f_{\gamma_{0}}\left(f_{i_{n}}-f_{i_{m}}\right)=0
$$

which further implies that $\left\|f_{\tau_{n}}-f_{\gamma_{m}}\right\|<1 / 2$ for $n, m \geqq N$. Let $C$ be the commutative $B^{*}$ algebra generated by $\left\{f_{\gamma_{n}}: n \geqq N\right\}$. We claim that spt $f_{\gamma_{N}} \subsetneq N\left(f_{\gamma_{N+2}}\right)$. If this is not true, then $\operatorname{spt} f_{\gamma_{N}}=\operatorname{spt} f_{\gamma_{N+1}}=\operatorname{spt} f_{\gamma_{N+2}}$ and so $f_{i_{N+1}}=f_{\gamma_{N+2}}=$ the characteristic function of $\operatorname{spt} f_{\gamma_{N}}$ by 3.2 , contradicting the choice of $\left\{f_{i_{n}}\right\}_{n=1}^{\infty}$. Thus $\exists x \in N\left(f_{i_{N+2}}\right) \backslash$ spt $f_{\gamma_{N}}$ which implies that $\left\|f_{7_{N+3}}(x)-f_{7_{N}}(x)\right\|=1$. This contradiction concludes the proof that a (nonabelian) $B^{*}$ algebra $A$ cannot have both a wellbehaved and a sham compact approximate identity.

It is is easy to give an example of a $\beta$ totally bounded approximate identity in $C_{0}(S)$ that is not canonical (and a fortiori, not wellbehaved). Our next result points out the rather interesting fact that in an abelian $B^{*}$ algebra a canonical chain totally bounded approximate identity is well-behaved.

Proposition 7.4. Let $\left\{e_{2}\right\}$ be a canonical chain totally bounded approximate identity for $C_{0}(S)$. Then $\left\{e_{\lambda}\right\}$ is well-behaved.

Proof. Let $\left\{\lambda_{n}\right\}$ be an increasing sequence in $\Lambda$ and $F=\bigcup_{n=1}^{\infty}$ spt $f_{\lambda_{n}}$. Then $F$ is clopen as in the proof of 3.3 and, for any compact subset $K$ of $F, K \subset N\left(e_{\lambda_{N}}\right)$ for some integer $N$, so that $e_{\lambda n} \equiv 1$ on $K$ for $n>N$. If $\lambda \in \Lambda$, let $K=\operatorname{spt} e_{\lambda} \cap F$ : then $e_{\lambda}\left(e_{\lambda_{n}}-e_{\lambda_{m}}\right)=0$ for $n$ and $m$ large enough by the preceding remarks. Therefore $\left\{e_{\lambda}\right\}$ is well-behaved.

Taylor [21] prove several interesting theorems about $M(A)$ assuming that the $B^{*}$ algebra $A$ has a well-behaved approximate identity. From 4.3 and 7.4 we see that (looking at the algebra generated by the approximate identity) an abelian, canonical, and chain totally bounded approximate identity for $A$ is a well-behaved approximate 
identity so Taylor's theorems hold in this case. We conjecture even more, viz., that if $A$ has a canonical chain totally bounded approximate identity, then the theorems in [21] hold. Our reason for believing this is the next proposition, which shows that a canonical chain totally bounded approximate identity is "almost" well-behaved.

Proposition 7.5. If $\left\{e_{\lambda}\right\}$ is a canonical chain totally bounded approximate identity in a Banach algebra $A$, then $\left\{e_{\lambda}\right\}$ satisfies the following condition: if $\varepsilon>0,\left\{\lambda_{n}\right\}$ is an increasing sequence in $\Lambda$, and $\lambda \in \Lambda$ there exists a positive integer $N$ so that $n, m>N$ implies

$$
\left\|e_{\lambda}\left(e_{\lambda_{n}}-e_{\lambda_{m}}\right)\right\|<\varepsilon \text {. }
$$

Proof. By chain total boundedness of $\left\{e_{\lambda}\right\}$, there is an integer $P$ so that for all positive integers $n$

$$
\min _{1 \leqq p \leqq P}\left\|e_{\lambda}\left(e_{\lambda_{n}}-e_{\lambda_{p}}\right)\right\|<\frac{\varepsilon}{2} .
$$

Choose $N \geqq P$ so that if $N<n<p, \exists q>p$ so that $\left\|e_{\lambda}\left(e_{\lambda_{n}}-e_{\lambda_{q}}\right)\right\|<\varepsilon$. If $n, m>N$ and $n<m$, choose $q>m$ so that $\left\|e_{\lambda}\left(e_{\lambda_{n}}-e_{\lambda_{q}}\right)\right\|<\varepsilon$. Then $\left\|e_{\lambda}\left(e_{\lambda_{n}}-e_{\lambda_{m}}\right)\right\|=\left\|e_{\lambda}\left(e_{\lambda_{n}}-e_{\lambda_{q}}\right) e_{\lambda_{m}}\right\| \leqq\left\|e_{\lambda}\left(e_{\lambda_{n}}-e_{\lambda_{q}}\right)\right\|<\varepsilon$.

EXAMPLE 7.6. We now give an example of an approximate identity that is well-behaved and not $\beta$ totally bounded. Let $R$ denote the real line and $A$ be the set of pairs $(i, j)$ where $i$ is any positive integer and $j=0$ or $j=1$. Order $A$ as follows:

(1) $(i, j)=\left(i^{\prime}, j^{\prime}\right)$ if $i=i^{\prime}$ and $j=j^{\prime}$;

(2) $(j, 0)>(i, 1)$ for all integers $i$ and $j$;

(3) $(i, 0)>(j, 0)$ if $i>j$.

If $\lambda=(i, 0)$ let $f_{\lambda}$ be in $C_{0}(R)$ so that $0 \leqq f_{\lambda} \leqq 1$ and $f_{\lambda} \equiv 1$ on $[-i, i]$ and $f_{\lambda} \equiv 0$ off $[-(i+1),(i+1)]$. If $\lambda=(i, 1)$, let $f_{\lambda}$ again be in $C_{0}(R)$ so that $0 \leqq f_{\lambda} \leqq 1, f_{\lambda}\left(x_{i}\right)=1$ where $x_{i}=1 / 2(1 /(i+1)+1 / i)$ and $f_{\lambda} \equiv 0$ off $[1 /(i+1), 1 / i]$. The net $\left\{f_{\lambda}\right\}$ is easily seen to be well-behaved but the infinite sequence $\{f(i, 1)\}$ is clealy not $\beta$ totally bounded.

EXAMPLE 7.7. In 3.3, we showed that if $C_{0}(S)$ has an approximate identity that is well-behaved (or $\beta$ totally bounded) then $S$ contains a clopen set $X$ so that $C_{0}(S)=B_{1} \oplus B_{2}$ where $B_{1}=\left\{f \in C_{0}(S) ; f \equiv 0\right.$ on $X\}$ and $B_{2}=\left\{f \in C_{0}(S): f \equiv 0\right.$ on $\left.S \backslash X\right\}$ are 2-sided ideals of $C_{0}(S)$. Obvious non-commutative generalizations of the above fail as we now show. Let $A$ be the algebra of compact operators on a Hilbert space $H,\left\{e_{j}: \gamma \in \Gamma\right\}$ an orthonormal basis for $H$, and $A$ the set of finite subsets of $\Gamma$ ordered by inclusion. If $\lambda \in \Lambda$, let $P_{\lambda}$ be the finite-dimensional projection defined by the equation 


$$
P_{\lambda}(h)=\sum_{\gamma \in \lambda}<h, e_{\gamma}>e_{\gamma} \text { for } h \in H .
$$

It is easy to show that $\left\{P_{\lambda}\right\}$ is a well-behaved and totally bounded approximate identity for $A$, but $A$ has no non-trivial decomposition as a direct sum of two-sided ideals [14].

REMARK 7.8. It is perhaps worth pointing out that if $C_{0}(S)$ and $C_{0}(T)$ have approximate identities with certain properties, so does $C_{0}(S X T)$ and the converse is also true. Suppose for example that $C_{0}(S)$ has a well-behaved approximate identity $\left\{e_{\lambda}\right\}$ and $C_{0}(T)$ has a well-behaved approximate identity $\left\{f_{\alpha}\right\}$. If $f$ and $g \in C_{0}(S\}$ and $C_{0}(T)$ respectively let $f \otimes g$ be the function on $S \times T$ defined by $f \otimes g(s, t)=f(s) g(t)$. It is easy to see that $f \otimes g \in C_{0}(S X T)$. Because the algebra generated by $\left\{f \otimes g \mid \begin{array}{l}f \in C_{0}(S) \\ g \in C_{0}(T)\end{array}\right\}$ is dense in $C_{0}(S X T)$ by the Stone-Weierstrass Theorem, the net $\left\{e_{\lambda} \otimes f_{\alpha}\right\}$ with directed set all pairs $(\lambda, \alpha)$ where $(\lambda, \alpha)>\left(\lambda^{\prime}, \alpha^{\prime}\right)$ if $\lambda>\lambda^{\prime}$ and $\alpha>\alpha^{\prime}$ is an approximate identity for $C_{0}(S X T)$ which is easily seen to be well-behaved. Conversely, if $\left\{e_{\lambda}\right\}$ is a well-behaved approximate identity for $C_{0}(S X T)$ and $t_{0} \in T$, the net of function $\left(f_{2}\right)$ defined by $f_{\lambda}(s)=e_{\lambda}\left(s, t_{0}\right)$ is a well behaved approximate identity for $C_{0}(S)$.

ExAMPLE 7.9. Our investigations of $\sigma\left(M(A), M(A)_{\beta}^{*}\right)$ relatively compact approximate identities is in the first stages only. We wish to present the following example, however, as it seems interesting. Let $S=$ the ordinals less than first uncountable with the order topology. $C_{0}(S)$ has no $\sigma\left(C_{b}(S), M(S)\right)$ relatively compact approximate identity. For, suppose that $C_{0}(S)$ has an approximate identity $\left\{e_{\lambda}\right\}$ which is $\sigma\left(C_{b}(S), M(S)\right.$ relatively compact. Note that $\left(\left|e_{\lambda}\right|^{2}\right)$ is an approximate identity which is also $\sigma\left(C_{b}(S), M(S)\right.$ ) relatively compact, so we may suppose $e_{\lambda} \geqq 0$. Let $\lambda_{1} \in \Lambda$ and $x_{1}=\min \left\{x \in S: y>x \Rightarrow e_{\lambda_{1}}(y)=0\right\}$. Choose $\lambda_{2} \in \Lambda$ so that $e_{\lambda_{2}}>2 / 3$ on $\left[0, x_{1}+1\right]$ and let $x_{2}=\min \{x \in S: y>x \Rightarrow \Rightarrow$ $e_{\lambda_{2}}(y)=0$. Note $x_{2} \geqq x_{1}+1$ so $x_{2}>x_{1}$.

Suppose $\lambda_{1}, \cdots, \lambda_{n}$ and $x_{1}, \cdots, x_{n}$ have been chosen so that:

(1) $e_{\lambda_{k}}>k /(k+1)$ on $\left[0, x_{k-1}+1\right]$ for $2 \leqq k \leqq n$

(2) $x_{k}=\min \left\{x \in S: y>x \Rightarrow e_{\lambda_{k}}(y)=0\right\}$

(3) $x_{n}>x_{n-1}>\cdots>x_{2}>x_{1}$.

By induction we select a sequence $\left(\lambda_{n}\right)$ in $\Lambda$ and a sequence $\left(x_{n}\right)$ from $X$ satisfying (1) and (2) and (3). Let $x=\operatorname{lub}\left\{x_{n}\right\}$. By assumption, $\exists f \in C_{b}(S)$ so that $e_{\lambda_{n}}$ clusters $\sigma\left(C_{b}(S), M(S)\right)$ to $f$. If $y>x$, $e_{\lambda_{n}}(y)=0$ for all $n$ so that $f(y)=0$. If $y<x$, then there is an integer $N$ so that $y<x_{n}$ for $n>N$ so that $e_{\lambda_{n}}(y)$ clusters to 1 ; therefore $f(y)=1$. We now show that $f$ cannot be continuous at $x$. Since $\left\{x_{n}\right\}$ is strictly increasing, $x_{n}<x$ for all $n$ so that $e_{\lambda_{n}}(x)=0$ for all 
$n$ and so $f(x)=0$; on the other hand, $x_{n} \rightarrow 2$, so, if $f$ were continuous, $f(x)$ would be the limit of the constant sequence $f\left(x_{n}\right)$, i.e. 1 . This contradiction concludes the proof that $C_{0}(S)$ has no $\sigma\left(C_{b}(S), M(S)\right.$ ) relatively compact approximate identity.

Our last result (7.10 below) answers only one of a number of questions of the following form: given an algebra $A$ with an approximate identity having property $P$ and another approximate identity $\left\{e_{\lambda}\right\}$, can we select from $\Lambda$ a subset $\Lambda_{0}$ (cofinal, perhaps) so that $\left\{e_{\lambda}: \lambda \in \Lambda_{0}\right\}$ has property $P$. Easy examples show that the subset $\Lambda_{0}$ in 7.10 need not be cofinal in $\Lambda$.

Proposition 7.10. If a Banach algebra $A$ has a countable approximate identity $\left\{f_{\gamma}\right\}$ and $\left\{e_{\lambda}\right\}$ is another approximate identity, then there is a countable subset $\Lambda_{0}$ of $\Lambda$ so that $\left\{e_{\lambda}: \lambda \in \Lambda_{0}\right\}$ is an approximate identity for $A$.

Proof. Choose a countable subset $\Lambda_{0}$ of $\Lambda$ so that $\lim _{\lambda \in \Lambda_{0}} e_{\lambda} f_{\gamma}=$ $\lim _{\lambda \in A_{0}} f_{i} e_{\lambda}=f_{i}$ for each $\gamma \in \Gamma$.

\section{REFERENCES}

1. C. A. Akemann, A Gelfand representation for arbitrary $C^{*}$-algebras, to appear.

2. - Interpolation in $W^{*}$-algebras, Duke Math. J., 35 (1968), 525-534.

3. W. G. Bade, Extensions of interpolating sets, Proceedings of a conference held at the University of California, Irvine, edited by B. R. Gelbaum, Thompson Book Company, Washington, 1967.

4. R. C. Buck, Bounded continuous functions on a locally compact space, Mich. Math. J., 5 (1958), 95-104.

5. R. C. Busby, Double centralizers and extensions of $C^{*}$-algebras, Trans. Amer. Math. Soc., 132 (1968), 79-99.

6. H. S. Collins and J. R. Dorroh, Remarks on certain function spaces, Math. Annalen, 176 (1968), 157-168.

7. J. B. Conway, The strict topology and compactness in the space of measures, Trans. Amer. Math. Soc., 126 (1967), 474-486.

8. - A theorem on sequential convergence of measures and some applications, Pacific J. Math., 28 (1969), 53-60.

9. M. M. Day, Normed linear spaces, Springer-Verlag, Berlin, 1958.

10. J. Dixmier. Les algebres d'opérateurs, dans l'éspace Hilbertien, Gauthier-Villars, Paris, 1957.

11. J. Dugundji, Topology, Allyn and Bacon, Boston, 1965.

12. I. Glicksberg, The representation of functionals by integrals, Duke Math., J., 19 (1952), 253-261.

13. J. Kelley, General Topology, Van Nostrand, 1955.

14. M. Naimark, Normed Rings, Groningen, The Netherlands: P. Noordhoff, 1960.

15. G. Pedersen, Measure theory for $C^{*}$-algebras, Math. Scand., 19 (1966), 131-145.

16. - Measure theory for $C^{*}$-algebras II, Math. Scand., 22 (1968), 63-74.

17. A. P. and W. J. Robertson, Topological vector spaces, Cambridge University Press, London, 1964. 
18. F. Sentilles and D. Taylor, Factorization in Banach algebras and the general strict topology, Trans. Amer. Math. Soc., 142 (1969), 141-152.

19. W. Summers, Factorization in Fréchet spaces, Studia Math., 39 (1971), 209-216.

20. D. Taylor, The strict topology for double centralizer algebras, Trans. Amer. Math. Soc., 150 (1970), 633-643.

21. — A general Phillips theorem for $C^{*}$-algebras and some applications, Pacific J. Math., 40 (1972), 477-488.

22. J. Wang, Multipliers of commutative Banach algebras, Pacific J. Math., 11 (1961), 1131-1149.

23. J. Webb, Sequential convergence in locally convex spaces, Proc, Camb. Phil. Soc., 64 (1968), 341-364.

Received June 1, 1971. These results appear in the dissertation of the second author above, written under the direction of the first author, and the work was partially supported by NSF grant GP-20866.

Louisiana State University 



\section{PACIFIC JOURNAL OF MATHEMATICS}

\section{EDITORS}

\section{H. SAMElson}

Stanford University

Stanford, California 94305

C. R. Новву

University of Washington

Seattle, Washington 98105
J. DugunduI

Department of Mathematics University of Southern California

Los Angeles, California 90007

RICHARD ARENS

University of California

Los Angeles, California 90024

\section{ASSOCIATE EDITORS}
E. F. BECKENBACH
B. H. NeUmanN
F. WOLF
K. YosHIDA

\section{SUPPORTING INSTITUTIONS}

UNIVERSITY OF BRITISH COLUMBIA

UNIVERSITY OF SOUTHERN CALIFORNIA

CALIFORNIA INSTITUTE OF TECHNOLOGY

STANFORD UNIVERSITY

UNIVERSITY OF CALIFORNIA

UNIVERSITY OF TOKYO

MONTANA STATE UNIVERSITY

UNIVERSITY OF UTAH

UNIVERSITY OF NEVADA

WASHINGTON STATE UNIVERSITY

NEW MEXICO STATE UNIVERSITY

OREGON STATE UNIVERSITY

UNIVERSITY OF OREGON

OSAKA UNIVERSITY

UNIVERSITY OF WASHINGTON

* *

AMERICAN MATHEMATICAL SOCIETY

NAVAL WEAPONS CENTER 


\section{Pacific Journal of Mathematics}

\section{Vol. 43, No. $1 \quad$ March, 1972}

Alexander (Smbat) Abian, The use of mitotic ordinals in cardinal

arithmetic ....................................... 1

Helen Elizabeth. Adams, Filtrations and valuations on rings ......... 7

Benno Artmann, Geometric aspects of primary lattices .............. 15

Marilyn Breen, Determining a polytope by Radon partitions ........... 27

David S. Browder, Derived algebras in $L_{1}$ of a compact group .......... 39

Aiden A. Bruen, Unimbeddable nets of small deficiency .............. 51

Michael Howard Clapp and Raymond Frank Dickman, Unicoherent

compactifications ............................... 55

Heron S. Collins and Robert A. Fontenot, Approximate identities and the strict topology ................................... 63

R. J. Gazik, Convergence in spaces of subsets................. 81

Joan Geramita, Automorphisms on cylindrical semigroups ........... 93

Kenneth R. Goodearl, Distributing tensor product over direct product ..... 107

Julien O. Hennefeld, The non-conjugacy of certain algebras of

operators ................................... 111

C. Ward Henson, The nonstandard hulls of a uniform space ........... 115

M. Jeanette Huebener, Complementation in the lattice of regular

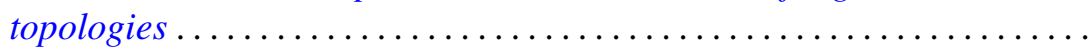

Dennis Lee Johnson, The diophantine problem $Y^{2}-X^{3}=A$ in a

polynomial ring .................................... 151

Albert Joseph Karam, Strong Lie ideals . . . . . . . . . . . . . . . . . . . . 157

Soon-Kyu Kim, On low dimensional minimal sets ............... 171

Thomas Latimer Kriete, III and Marvin Rosenblum, A Phragmén-Lindelöf

theorem with applications to $M(u, v)$ functions ..... . .

William A. Lampe, Notes on related structures of a universal algebra . . . . 189

Theodore Windle Palmer, The reducing ideal is a radical .

207

Kulumani M. Rangaswamy and N. Vanaja, Quasi projectives in abelian and module categories ................................ 221

Ghulam M. Shah, On the univalence of some analytic functions ......... 239

Joseph Earl Valentine and Stanley G. Wayment, Criteria for Banach

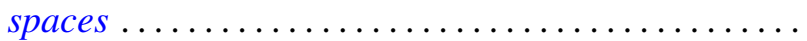

Jerry Eugene Vaughan, Linearly stratifiable spaces ............... 253

Zbigniew Zielezny, On spaces of distributions strongly regular with respect to partial differential operators ..................... 